\title{
Phase diagrams and electronic properties of B-S and H-B-S systems under high pressure
}

\author{
Xin Du, ${ }^{1}$ Shoutao Zhang $\odot,{ }^{1}$ Jianyan Lin, ${ }^{1}$ Xiaohua Zhang, ${ }^{1}$ Aitor Bergara, $, 23,4,{ }^{*}$ and Guochun Yang $\oplus^{1, \dagger}$ \\ ${ }^{1}$ Centre for Advanced Optoelectronic Functional Materials Research and Key Laboratory for UV Light-Emitting Materials \\ and Technology of Northeast Normal University, Changchun 130024, China \\ ${ }^{2}$ Departamento de Física de la Materia Condensada, Universidad del País Vasco-Euskal Herriko Unibertsitatea, \\ UPV/EHU, 48080 Bilbao, Spain \\ ${ }^{3}$ Donostia International Physics Center (DIPC), 20018 Donostia, Spain \\ ${ }^{4}$ Centro de Física de Materiales CFM, Centro Mixto CSIC-UPV/EHU, 20018 Donostia, Spain
}

(Received 15 July 2019; revised manuscript received 4 October 2019; published 29 October 2019)

\begin{abstract}
Pressure has become an effective way to obtain new materials with desirable properties. Considering the various stoichiometries, wide applications, and unique structures of binary H-S, H-B, and B-S compounds, especially at high pressures, it is worth expecting that they might form ternary compounds with interesting properties as well. Here, phase stabilities of H-B-S ternary compounds, in the pressure range from 0 to $200 \mathrm{GPa}$, are reliably determined through first-principles unbiased structural search calculations. A hitherto unknown orthorhombic HBS compound with Ama2 symmetry is identified to be stable above $25 \mathrm{GPa}$, in which $\mathrm{B}$ and $\mathrm{S}$ atoms alternate to form distorted chains and $\mathrm{H}$ atoms are covalently bonded with Bs. With pressure, Ama2 HBS undergoes a semiconductor-to-metal electronic transition and even becomes superconducting. The exploration of the binary B-S phase diagram indicates that B-S compounds tend to decompose into elemental solids above $208 \mathrm{GPa}$. However, two $S$-rich phases, $\mathrm{B}_{2} \mathrm{~S}_{3}$ and $\mathrm{BS}_{2}$, are predicted at low pressures, exhibiting semiconducting and metallic properties, respectively. $\mathrm{BS}_{2}$ shows a calculated $T_{c}$ value of $21.9 \mathrm{~K}$ at $200 \mathrm{GPa}$, becoming a superconductor among bulk binary B-S compounds.
\end{abstract}

DOI: 10.1103/PhysRevB.100.134110

\section{INTRODUCTION}

One of the most important challenges in condensed matter physics and materials science is the prediction of hightemperature superconductors [1,2]. Among the various superconducting materials, high-pressure stable hydrogen-rich compounds have become the main candidates to superconductor at room temperature due to the strong electron-phonon coupling and light atomic mass of hydrogen [3-7]. Recently, the synergy between experimental and theoretical studies has again broken the record of superconducting transition temperatures, reigniting a new wave in the research of $\mathrm{H}$-rich compounds [8-11].

Up to now, most studies were focused on binary hydrides [12-20]. The recent progress on this theme can be found in review articles [21-27]. However, ternary hydrides might also be potential high-temperature superconductors [28-33], and some studies have shown that they not only show high superconducting transition temperatures $\left(T_{c}\right)$ but also exhibit unexpected superconducting mechanisms. For example, the predicted ternary hydride $\mathrm{MgSiH}_{6}$ has a $T_{c}$ value of $63 \mathrm{~K}$ at $250 \mathrm{GPa}$, and its superconducting transition mainly comes from phonon softenings along $\Gamma \rightarrow \mathrm{X}$ and $\Gamma \rightarrow \mathrm{M}$ directions [34]. $\mathrm{H}_{6} \mathrm{SSe}$, obtained from $\mathrm{H}_{3} \mathrm{~S}$ with half of its $\mathrm{S}$ atoms replaced by Se, presents a lower $T_{c}$ value than that of $\mathrm{H}_{3} \mathrm{~S}$, indicating that a strong covalent bonding is a dominant factor in

\footnotetext{
*Corresponding author: a.bergara@ehu.eus

$\dagger$ Corresponding author: yanggc468@ nenu.edu.cn
}

these superconducting materials [35]. Pressure-induced stable $\mathrm{H}_{3} \mathrm{SXe}$ might also become a high-temperature superconductor, where Xe facilitates the hydrogen-bond symmetrization [36]. Additionally, several ternary hydrides, such as $\mathrm{Fe}_{2} \mathrm{SH}_{3}$ [37], $\mathrm{BaReH}_{9}$ [38], $\mathrm{KAuH}_{2}$ [39], $\mathrm{CaYH}_{12}$ [30], and $\mathrm{MgCH}_{4}$ [40], also show superconductivity.

It is well accepted that pressure favors the stabilization of unusual stoichiometric compounds [41-44], exhibiting interesting physical and chemical properties. For example, several stable sulfur hydrides (e.g., $\mathrm{H}_{2} \mathrm{~S}_{3}, \mathrm{H}_{3} \mathrm{~S}_{2}, \mathrm{HS}_{2}, \mathrm{H}_{3} \mathrm{~S}$, and $\mathrm{H}_{4} \mathrm{~S}_{3}$ ) [45-47] have been identified at high pressures. More interestingly, $\mathrm{H}_{3} \mathrm{~S}$ shows a remarkably high $T_{c}$ value of $203 \mathrm{~K}$ [48]. On the other hand, boron (B) and $\mathrm{H}$ can form stable binary compounds at both ambient and high pressures. $\mathrm{B}_{2} \mathrm{H}_{6}$ is a highly reactive and versatile reagent and a high-energydensity material at ambient pressure which metallizes and even becomes a superconductor under high pressure [49-55]. Extensive efforts have been made to investigate their highpressure phase diagram [56,57]. Thus far, two stable boron hydrides have been found (e.g., $\mathrm{BH}$ and $\mathrm{BH}_{2}$ ). The semiconducting Ibam BH phase transforms into the metallic $P 6 / \mathrm{mmm}$ structure, which also becomes a superconductor with a $T_{c}$ of $14.1 \sim 21.4 \mathrm{~K}$ at $168 \mathrm{GPa}$ [56]. For B-S compounds, most of the current studies are focused on structures and electronic properties at ambient pressure [58-62]. Structural phase transitions of $\mathrm{BS}$ and $\mathrm{B}_{2} \mathrm{~S}_{3}$ have been studied below $50 \mathrm{GPa}$ [63-65]. Thus far, their high-pressure phase diagram with a wide range of chemical compositions has not been fully determined yet. 
Considering the rich structures, phase stabilities, and diverse properties of $\mathrm{H}-\mathrm{S}, \mathrm{H}-\mathrm{B}$, and B-S binary systems, it may be worth studying H-B-S ternary compounds under high pressure. In this work, to reliably determine the phase diagram of H-B-S ternary compounds, we first explore the stable B-S compounds with various $\mathrm{B}_{x} \mathrm{~S}_{y}(x$ and $y=1-5 ; x=1-5$ and $y=1 ; x=2-3$ and $y=3-2$ ) compositions at high pressures through first-principles swarm-intelligence structural calculations $[66,67]$. Then, candidate structures of $\mathrm{H}-\mathrm{B}-\mathrm{S}$ ternary compounds with $\mathrm{H}_{x} \mathrm{~B}_{y} \mathrm{~S}_{z}(x=1-10 ; y=1-2 ; z=$ $1-2$ ) compositions are considered to build the ternary phase diagram. There is just one stable chemical composition in $\mathrm{H}$ $\mathrm{B}-\mathrm{S}$ under high pressure. The semiconducting orthorhombic HBS is found to be stable at $25 \mathrm{GPa}$, which becomes metallic at $\sim 200 \mathrm{GPa}$ and appears to be superconducting at $300 \mathrm{GPa}$. Stable binary B-S compounds become unstable with respect to elemental solids at very high pressures. Interestingly, the newly identified $\mathrm{BS}_{2}$ compound becomes a superconductor with a $T_{c}$ of $21.9 \mathrm{~K}$ at $200 \mathrm{GPa}$. Our work provides an opportunity to understand the structures and properties of $\mathrm{H}-\mathrm{B}-\mathrm{S}$ and B-S systems at high pressures.

\section{COMPUTATIONAL DETAILS}

First-principles-based structural prediction technology plays an important role in the discovery of new materials, especially at high pressures [66,68-70]. In order to reliably determine the phase stability, finding adequate candidates is a necessary prerequisite. Our structure searches of $\mathrm{H}-\mathrm{B}-\mathrm{S}$ and B-S systems were performed with the CALYPSO prediction method based on swarm intelligence [67]. CALYPSO is able to find the most stable structure by just knowing the chemical composition, whose effectiveness has been confirmed in various kinds of compounds [37,45,71-74]. Specific details of the calculations are given in the Supplemental Material [75].

Calculations of the structural relaxations and electronic properties were carried out using density functional theory (DFT) [76,77] as implemented in the Vienna ab initio Simulation Package (VASP) code [78]. The exchange-correlation energy was treated within the Perdew-Burke-Ernzerhof (PBE) [79] generalized gradient approximation (GGA) [80]. The adopted all-electron projector augmented-wave (PAW) [81] pseudopotentials of $\mathrm{B}, \mathrm{S}$, and $\mathrm{H}$ treat $2 s^{2} 2 p^{1}, 3 s^{2} 3 p^{4}$, and $1 s^{1}$ electrons as the valence electrons. To obtain a better energy convergence, we have chosen a plane-wave cutoff energy of $800 \mathrm{eV}$ and Monkhorst-Pack $k$ meshes [82] of $2 \pi \times 0.03 \AA^{-1}$. Phonon dispersion calculations, as implemented in the PHONOPY code [83], were used to determine the dynamic stability of thermodynamically stable phases. The electron-phonon coupling for superconducting properties of stable metal phases are performed within the framework of the linear-response theory via the Quantum ESPRESSO package [84].

\section{RESULTS AND DISCUSSION}

\section{A. Phase stability}

To reliably determine the phase stability of ternary compounds at high pressures, we need to know the stable structures of the corresponding binary compounds or elemental solids. As discussed above, the high-pressure phase diagrams of binary $\mathrm{B}-\mathrm{H}$ and $\mathrm{S}-\mathrm{H}$ compounds have been extensively studied, but the binary phase stability of B$\mathrm{S}$ compounds at high pressures has not been explored. First, we perform an extensive structure search to find the most stable structures for each $\mathrm{B}_{x} \mathrm{~S}_{y}(x=1$ and $y=1-$ $5 ; x=1-5$ and $y=1 ; x=2-3$ and $y=3-2$ ) composition at $0 \mathrm{~K}$ and selected pressures of 1 atm and $25,50,100$, and $200 \mathrm{GPa}$ via first-principles swarmintelligence search calculations. The calculated convex hull is shown in Fig. S1 (see Supplemental Material [75]). Besides reproducing the already known structures, we find another two S-rich phases of $\mathrm{B}_{2} \mathrm{~S}_{3}$ and $\mathrm{BS}_{2}$. Notably, B$\mathrm{S}$ compounds, stabilized at atmospheric and low pressures, tend to decompose with respect to elemental solids above $208 \mathrm{GPa}$.

Considering that H-rich compounds might show high superconducting transition temperatures, we have mainly focused in the structural prediction of H-rich compositions of $\mathrm{H}_{x} \mathrm{~B}_{y} \mathrm{~S}_{z}(x=1-10 ; y=1-2 ; z=1-2)$ at 25, 50, 100, and $200 \mathrm{GPa}$. Then, we have chosen the structure with the
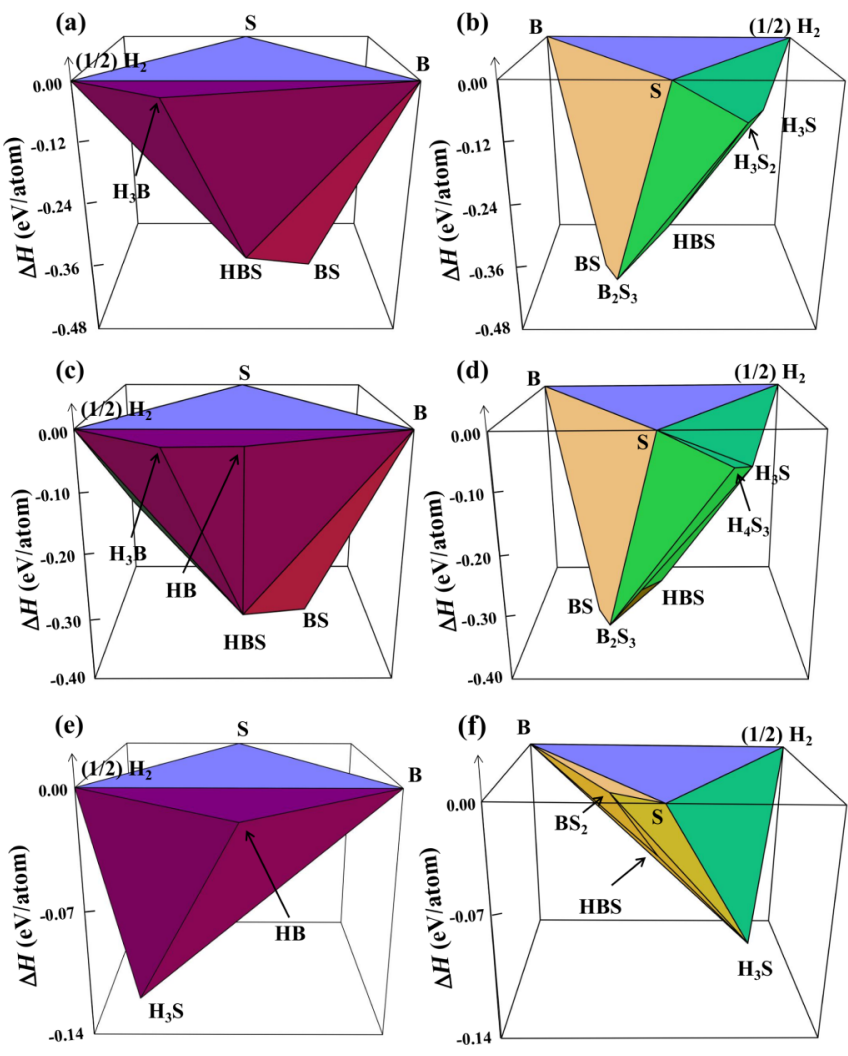

FIG. 1. Phase diagram of H-B-S system at (a), (b) $25 \mathrm{GPa}$, (c), (d) $50 \mathrm{GPa}$, and (e), (f) $200 \mathrm{GPa}$. At $25 \mathrm{GPa}, P-1 \mathrm{H}_{3} \mathrm{~B}$ [50], $C 2 / c \mathrm{H}_{3} \mathrm{~S}$ [46], $P 2_{1} \mathrm{H}_{3} \mathrm{~S}_{2}$ [47], $R-3 m$ BS [57], $C c \mathrm{~B}_{2} \mathrm{~S}_{3}, P 6_{3} / m(1 / 2) \mathrm{H}_{2}$ [86], Pnnm for B [87], and $I 4_{1} /$ acd for $\mathrm{S}$ [88] are used to determine the phase stability. On the other hand, at $50 \mathrm{GPa}$, Ibam $\mathrm{HB}, P 2_{1} / c \mathrm{H}_{3} \mathrm{~B}$ [56], $C 2 / c \mathrm{H}_{3} \mathrm{~S}$ [46], $P 2_{1} 2_{1} 2_{1} \mathrm{H}_{4} \mathrm{~S}_{3}$ [46], $C c \mathrm{~B}_{2} \mathrm{~S}_{3}, P-3 m 1$ BS [64], $P 6_{3} / m$ for $(1 / 2) \mathrm{H}_{2}, P n n m$ for B [56], and $I 4_{1} /$ acd for $\mathrm{S}$ [88] are chosen. Finally, at $200 \mathrm{GPa}, P 6 / \mathrm{mmm} \mathrm{HB}$ [56], Im-3m $\mathrm{H}_{3} \mathrm{~S}$ [46], $C 2 / c$ for $(1 / 2) \mathrm{H}_{2}, C m c a$ for B [56], and $R-3 m$ for $\mathrm{S}$ [89] are selected. 

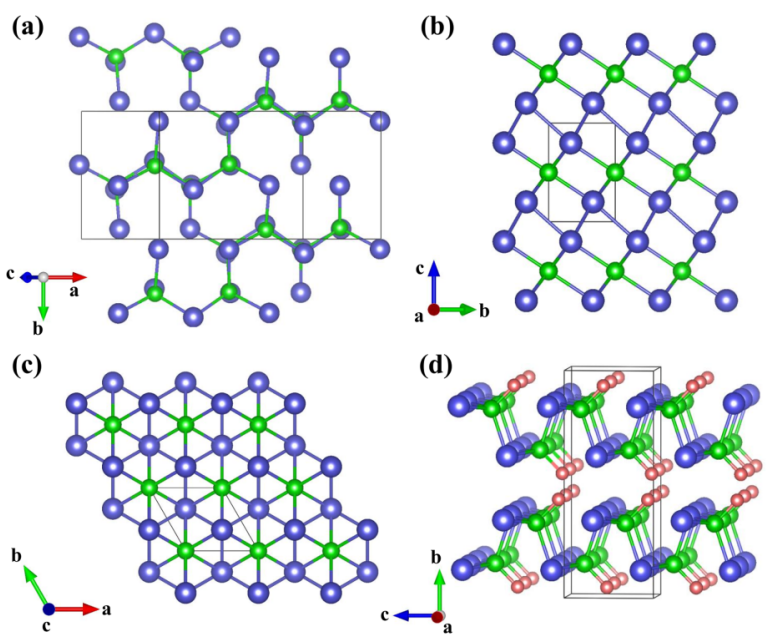

(e)
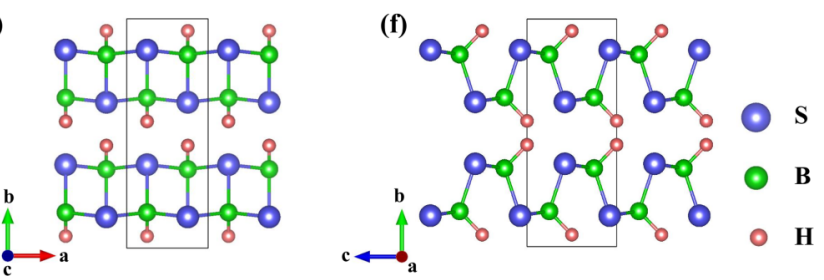

FIG. 2. Crystal structures of the stable compounds: (a) $C c \mathrm{~B}_{2} \mathrm{~S}_{3}$ at $50 \mathrm{GPa}$, (b), (c) $P-3 m 1 \mathrm{BS}_{2}$ at $200 \mathrm{GPa}$, and (d), (e), (f) $A m a 2$ HBS at $100 \mathrm{GPa}$. Pink, green, and purple spheres represent $\mathrm{H}, \mathrm{B}$, and $\mathrm{S}$ atoms, respectively.

lower enthalpy to determine the relative phase stability. In general, for ternary compounds, the structure with a negative formation enthalpy with respect to binary compounds, elemental solids, or their combinations becomes a potential candidate for experimental synthesis. The calculated convex hulls for H-B-S compounds are shown in Fig. 1, where the phases located at the edges of the polyhedron are thermodynamically stable [85]. In the pressure range we are considering, HBS is the only stable ternary compound. The absence of any imaginary frequencies in the calculated phonon dispersion curves of $C c \mathrm{~B}_{2} \mathrm{~S}_{3}, P-3 m 1 \mathrm{BS}_{2}$, and Ama2 HBS indicate they are dynamically stable (Fig. S2 [75]).

\section{B. Structural geometry}

Previous studies found two stable phases for $\mathrm{B}_{2} \mathrm{~S}_{3}[61,63]$ with $P 2_{1} / c$ and $I 4_{1} / a$ symmetries. However, according to our structural predictions, another $\mathrm{B}_{2} \mathrm{~S}_{3}$ phase [space group $C c$, four formula units per cell; Fig. 2(a)] becomes stable above $15 \mathrm{GPa}$. In $C c \mathrm{~B}_{2} \mathrm{~S}_{3}$ each $\mathrm{B}$ atom is four-coordinated, whereas $\mathrm{S}$ atoms have two different coordination environments: one is two-coordinated while the other is three-coordinated. The basic building block is a $\mathrm{B}_{3} \mathrm{~S}_{3}$ six-membered ring with an alternated arrangement of B and $\mathrm{S}$ atoms, which has also been observed in the other two low-pressure phases (Fig. S3 [75]). Cc $\mathrm{B}_{2} \mathrm{~S}_{3}$ decomposes into $\mathrm{BS}$ and $\mathrm{S}$ above $95 \mathrm{GPa}$ (Fig. S4 [75]). $P 2_{1} / c \mathrm{BS}_{2}$ is already stable at ambient pressure [58] but decomposes with pressure (Fig. S5 [75]). However, according to our structural search, $\mathrm{BS}_{2}$ becomes stable again at the pressure range of $170 \sim 208 \mathrm{GPa}$ [space group $P-3 m 1,1$ formula unit per cell; Figs. 2(b) and 2(c)]. In $P-3 m 1 \mathrm{BS}_{2}$ each B atom is six-coordinated with $\mathrm{S}$ atoms, whereas $\mathrm{S}$ atoms are threecoordinated both with $\mathrm{B}$ and $\mathrm{S}$, forming an interconnected zigzaglike $\mathrm{S}$ atom layer with an $\mathrm{S}-\mathrm{S}$ distance of $2.06 \AA$, which is shorter than $\mathrm{S}_{2}{ }^{2-}(2.12 \AA)$ in $\mathrm{Li}_{2} \mathrm{~S}_{2}$ [90]. HBS stabilizes into an orthorhombic structure [space group Ama2, four formula units per cell; Fig. 2(d)] and has no phase transitions in the considered pressure range. The most striking feature is that $\mathrm{B}$ and $\mathrm{S}$ atoms alternate to form a distorted chain along the $c$ direction, in which $\mathrm{H}$ atoms attach to $\mathrm{B}$ atoms. These chains are crosslinked through B-S bonds in the $a c$ plane, showing a typical layered structure. Moreover, the above-mentioned alternated arrangement of $\mathrm{B}_{3} \mathrm{~S}_{3}$ six-membered rings is also observed.
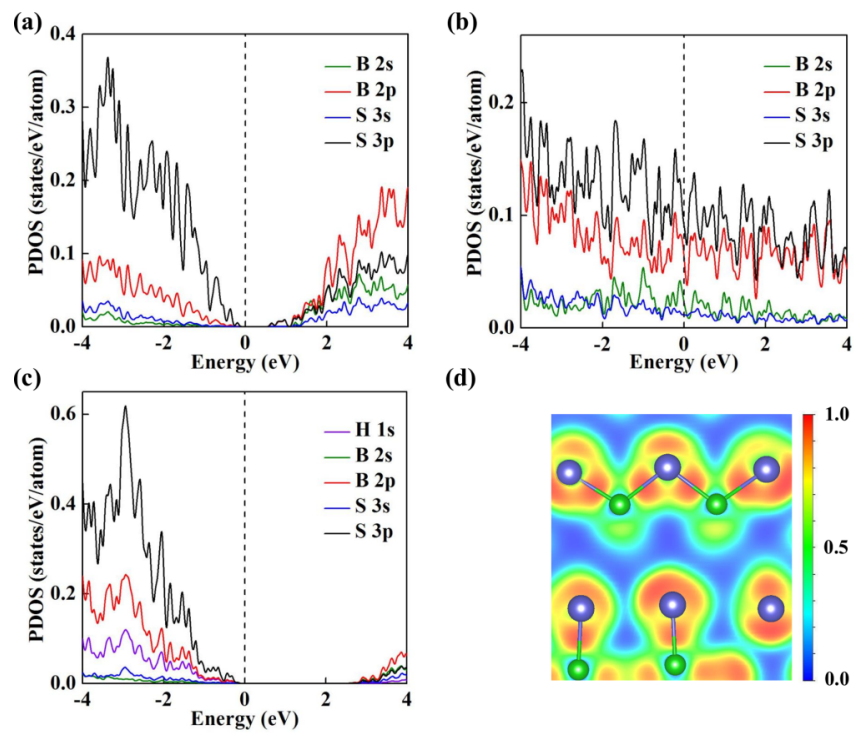

(e)

(d)

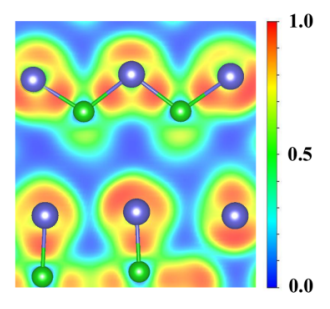

(f)
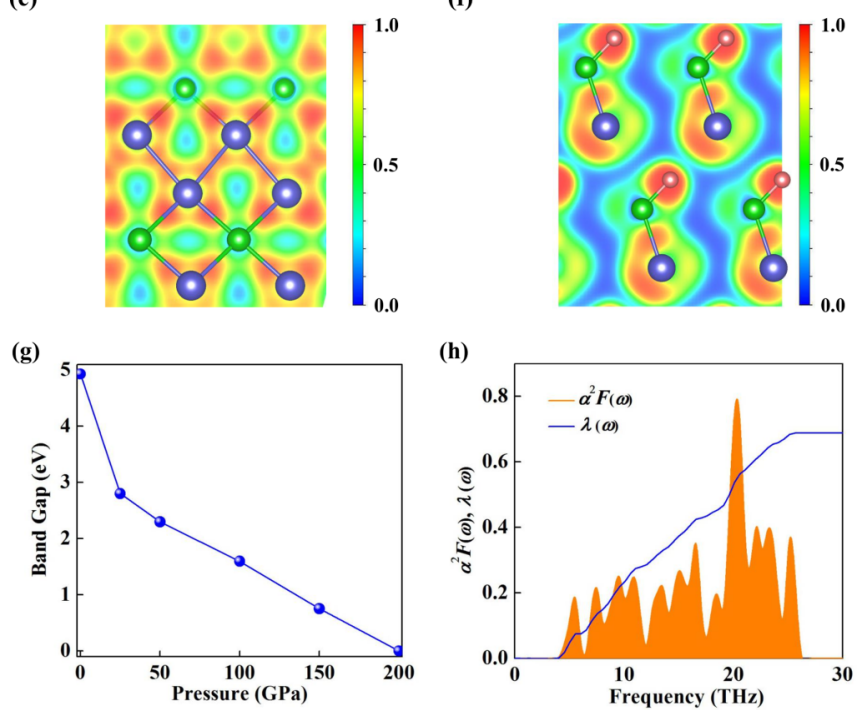

FIG. 3. Projected density of states (PDOS) within the HSE06 hybrid functional of (a) $C c \mathrm{~B}_{2} \mathrm{~S}_{3}$, (b) $P-3 m 1 \mathrm{BS}_{2}$, and (c) $A m a 2$ HBS at 50, 200, and $25 \mathrm{GPa}$, respectively. The vertical dashed line represents the Fermi level. (d), (e), (f) The ELF of $\mathrm{B}_{2} \mathrm{~S}_{3}, \mathrm{BS}_{2}$, and HBS, respectively. (g) The calculated band gap of Ama2 HBS as a function of pressure from 0 to $200 \mathrm{GPa}$. (h) The Eliashberg spectral function $\alpha^{2} F(\omega)$ and integrated electron-phonon coupling parameters $\lambda(\omega)$ of $P-3 m 1 \mathrm{BS}_{2}$ at $200 \mathrm{GPa}$. 


\section{Electronic properties}

To reliably determine the electronic properties, we have chosen the Heyd-Scuseria-Ernzerhof (HSE06) [91,92] hybrid functional to calculate the electronic band structures and partial density of states (PDOS) of $\mathrm{B}_{2} \mathrm{~S}_{3}, \mathrm{BS}_{2}$, and HBS [Fig. S6 [75] and Figs. 3(a)-3(c)]. Both $B_{2} S_{3}$ and HBS show indirectband-gap semiconducting character with band-gap values of 0.8 and $2.8 \mathrm{eV}$, respectively, whereas $\mathrm{BS}_{2}$ is metallic. For $\mathrm{B}_{2} \mathrm{~S}_{3}$ and $\mathrm{BS}_{2}$, there is a strong overlap between $\mathrm{S} 2 p$ and B $2 p$ orbitals below the Fermi level [Figs. 3(a) and 3(b)], indicating the formation of a strong chemical bonding. For $\mathrm{BS}_{2}$, the flatband analysis shows that the $\mathrm{S} 3 p$ orbital makes the major contribution to the Fermi level (Fig. S7 [75]). The analysis of the electronic localization function (ELF) [93] further confirms that B-S and S-S bonds are covalent [Figs. 3(d) and 3(e)]. Bader charge analysis shows charge transfer from $\mathrm{B}$ to $\mathrm{S}$, indicating that $\mathrm{B}-\mathrm{S}$ bonds are polar covalent (Table S2 [75]) [94]. The PDOS of HBS exhibits there is a strong hybridization between $\mathrm{S} 2 p$ and B $2 p$, and $\mathrm{H} 1 s$ and $\mathrm{B} 2 p$ or $2 s$ states [Fig. 3(c)], which are also covalent bondings [Fig. 3(f)]. The pressure-dependent electronic properties show the band gap of HBS gradually decreases and eventually closes, becoming a metal at $200 \mathrm{GPa}$ [Fig. 3(g)].

Considering the already known superconductivity of binary $\mathrm{H}-\mathrm{S}$ and $\mathrm{H}-\mathrm{B}$ compounds and the pressure-induced metallization of $\mathrm{BS}_{2}$ and HBS, we have also explored their eventual superconducting transitions. Surprisingly, the calculated superconducting transition temperature $\left(T_{c}\right)$ of $\mathrm{BS}_{2}$ is $21.9 \mathrm{~K}$ at $200 \mathrm{GPa}$, which is higher than $9.3 \mathrm{~K}$ of $\mathrm{HS}_{2}$ [47]. The analysis of the projected phonon density of states indicates that frequency vibrations mainly originate from the coupling between B and S atoms (Fig. S8 [75]). The integrated electron-phonon coupling parameter $\lambda(\omega)$ and Eliashberg spectral function $\alpha^{2} F(\omega)$ are shown in Fig. 3(h). The calculated $\lambda$ is 0.69 at $200 \mathrm{GPa}$ and is much lower than the 2.19 of $\mathrm{H}_{3} \mathrm{~S}$ [95]. For HBS, it shows no superconductivity at $200 \mathrm{GPa}$ but becomes superconducting at 300 and $400 \mathrm{GPa}$ with $T_{c}$ of 0.8 and $1.9 \mathrm{~K}$, respectively. The analysis of the projected phonon density of states indicates that the lowfrequency vibrations $(<15 \mathrm{THz})$ mainly originate from the coupling between B and S atoms; the high-frequency modes are associated with $\mathrm{H}$ atoms, while the middle-frequency stretching modes come from the coupling between B and H (Fig. S8 [75]). The integrated electron-phonon coupling parameter $\lambda(\omega)$ and Eliashberg spectral function $\alpha^{2} F(\omega)$ are shown in Fig. S8 [75]. The calculated $\lambda$ is only 0.27 at 300 $\mathrm{GPa}$, and the low-frequency translational vibration $(<42 \mathrm{THz})$ modes give the main contribution (95\%) to $\lambda$.

\section{CONCLUSIONS}

In summary, high-pressure phase diagrams of B-S and $\mathrm{H}-$ B-S systems are reliably built through first-principles swarmintelligence structural search calculations. A semiconducting $\mathrm{B}_{2} \mathrm{~S}_{3}$ phase is identified above $15 \mathrm{GPa}$. The identified $P-3 m 1$ $\mathrm{BS}_{2}$ compound is a superconductor in the bulk binary B-S compounds, having a $T_{c}$ of $21.9 \mathrm{~K}$ at $200 \mathrm{GPa}$. For ternary H-B-S systems, the only stable compound under pressure is HBS, exhibiting diverse pressure-related electronic properties (i.e., semiconductor $\rightarrow$ metallizatic $\rightarrow$ superconductor). Chemical bonding analysis reveals that the structural stability of HBS and B-S compounds is mainly attributed to the strong $\mathrm{B}-\mathrm{S}$ covalent bonds.

\section{ACKNOWLEDGMENTS}

The authors acknowledge funding support from the Natural Science Foundation of China under Grants No. 21573037, No. 21873017, No. 11704062, and No. 51732003, the Postdoctoral Science Foundation of China under Grant No. 2013M541283, the Natural Science Foundation of Jilin Province (Grant No. 20190201231JC), the "111" Project (No. B13013), and the Fundamental Research Funds for the Central Universities (2412017QD006). The work was carried out at National Supercomputer Center in Tianjin, and the calculations were performed on TianHe-1 (A). A.B. acknowledges financial support from the Spanish Ministry of Economy and Competitiveness (FIS2016-76617-P) and the Department of Education, Universities and Research of the Basque Government and the University of the Basque Country (IT756-13).
[1] H. Wang, X. Li, G. Gao, Y. Li, and Y. Ma, Wiley Interdiscip. Rev.: Comput. Mol. Sci. 8, e1330 (2018).

[2] H.-K. Mao, X.-J. Chen, Y. Ding, B. Li, and L. Wang, Rev. Mod. Phys. 90, 015007 (2018).

[3] J. S. Tse, Y. Yao, and K. Tanaka, Phys. Rev. Lett. 98, 117004 (2007).

[4] A. Shamp, T. Terpstra, T. Bi, Z. Falls, P. Avery, and E. Zurek, J. Am. Chem. Soc. 138, 1884 (2016).

[5] V. V. Struzhkin, D. Y. Kim, E. Stavrou, T. Muramatsu, H.-k. Mao, C. J. Pickard, R. J. Needs, V. B. Prakapenka, and A. F. Goncharov, Nat. Commun. 7, 12267 (2016).

[6] X.-J. Chen, J.-L. Wang, V. V. Struzhkin, H.-k. Mao, R. J. Hemley, and H.-Q. Lin, Phys. Rev. Lett. 101, 077002 (2008).

[7] Y. Li, G. Gao, Y. Xie, Y. Ma, T. Cui, and G. Zou, Proc. Natl. Acad. Sci. USA 107, 15708 (2010).
[8] H. Liu, I. I. Naumov, R. Hoffmann, N. W. Ashcroft, and R. J. Hemley, Proc. Natl. Acad. Sci. USA 114, 6990 (2017).

[9] F. Peng, Y. Sun, C. J. Pickard, R. J. Needs, Q. Wu, and Y. Ma, Phys. Rev. Lett. 119, 107001 (2017).

[10] A. P. Drozdov, P. P. Kong, V. S. Minkov, S. P. Besedin, M. A. Kuzovnikov, S. Mozaffari, L. Balicas, F. F. Balakirev, D. E. Graf, V. B. Prakapenka et al., Nature (London) 569, 528 (2019).

[11] M. Somayazulu, M. Ahart, A. K. Mishra, Z. M. Geballe, M. Baldini, Y. Meng, V. V. Struzhkin, and R. J. Hemley, Phys. Rev. Lett. 122, 027001 (2019).

[12] E. S. Wiedner, M. B. Chambers, C. L. Pitman, R. M. Bullock, A. J. M. Miller, and A. M. Appel, Chem. Rev. 116, 8655 (2016).

[13] K. Abe, Phys. Rev. B 96, 144108 (2017). 
[14] T. Scheler, M. Marqués, Z. Konôpková, C. L. Guillaume, R. T. Howie, and E. Gregoryanz, Phys. Rev. Lett. 111, 215503 (2013).

[15] B. Li, Y. Ding, D. Y. Kim, R. Ahuja, G. Zou, and H.-K. Mao, Proc. Natl. Acad. Sci. USA 108, 18618 (2011).

[16] J. Ying, X. Li, E. Greenberg, V. B. Prakapenka, H. Liu, and V. V. Struzhkin, Phys. Rev. B 99, 224504 (2019).

[17] T. Scheler, F. Peng, C. L. Guillaume, R. T. Howie, Y. Ma, and E. Gregoryanz, Phys. Rev. B 87, 184117 (2013).

[18] G. Gao, R. Hoffmann, N. W. Ashcroft, H. Liu, A. Bergara, and Y. Ma, Phys. Rev. B 88, 184104 (2013).

[19] S. Yu, X. Jia, G. Frapper, D. Li, A. R. Oganov, Q. Zeng, and L. Zhang, Sci. Rep. 5, 17764 (2015).

[20] M. A. Kuzovnikov and M. Tkacz, Phys. Rev. B 93, 064103 (2016).

[21] E. Zurek and T. Bi, J. Chem. Phys. 150, 050901 (2019).

[22] E. Zurek, Comments Inorg. Chem. 37, 78 (2017).

[23] H.-Y. Lv, M. Chen, Y. Feng, W.-J. Li, G.-H. Zhong, and C.-L. Yang, J. Chin. Chem. Soc. 66, 1246 (2019).

[24] D. Duan, Y. Liu, Y. Ma, Z. Shao, B. Liu, and T. Cui, Natl. Sci. Rev. 4, 121 (2017).

[25] J. A. Flores-Livas, L. Boeri, A. Sanna, G. Profeta, R. Arita, and M. Eremets, arXiv:1905.06693.

[26] T. Bi, N. Zarifi, T. Terpstra, and E. Zurek, Reference Module in Chemistry, Molecular Sciences and Chemical Engineering (2019), doi: 10.1016/B978-0-12-409547-2.11435-0.

[27] D. V. Semenok, I. A. Kruglov, I. A. Savkin, A. G. Kvashnin, and A. R. Oganov, arXiv:1806.00865.

[28] M. A. Olea-Amezcua, O. De la Peña-Seaman, and R. Heid, Phys. Rev. B 99, 214504 (2019).

[29] C. Zhang, G. Sun, J. Wang, C. Lu, Y. Jin, X. Kuang, and A. Hermann, ACS Appl. Mater. Interfaces 9, 26169 (2017).

[30] X. Liang, A. Bergara, L. Wang, B. Wen, Z. Zhao, X.-F. Zhou, J. He, G. Gao, and Y. Tian, Phys. Rev. B 99, 100505(R) (2019).

[31] D. Meng, M. Sakata, K. Shimizu, Y. Iijima, H. Saitoh, T. Sato, S. Takagi, and S.-i. Orimo, Phys. Rev. B 99, 024508 (2019).

[32] Y. Ma, D. Duan, Z. Shao, D. Li, L. Wang, H. Yu, F. Tian, H. Xie, B. Liu, and T. Cui, Phys. Chem. Chem. Phys. 19, 27406 (2017).

[33] Y. Sun, J. Lv, Y. Xie, H. Liu, and Y. Ma, Phys. Rev. Lett. 123, 097001 (2019).

[34] Y. Ma, D. Duan, Z. Shao, H. Yu, H. Liu, F. Tian, X. Huang, D. Li, B. Liu, and T. Cui, Phys. Rev. B 96, 144518 (2017).

[35] B. Liu, W. Cui, J. Shi, L. Zhu, J. Chen, S. Lin, R. Su, J. Ma, K. Yang, M. Xu et al., Phys. Rev. B 98, 174101 (2018).

[36] D. Li, Y. Liu, F.-B. Tian, S.-L. Wei, Z. Liu, D.-F. Duan, B.-B. Liu, and T. Cui, Frontiers Phys. 13, 137107 (2018).

[37] S. Zhang, L. Zhu, H. Liu, and G. Yang, Inorg. Chem. 55, 11434 (2016).

[38] T. Muramatsu, W. K. Wanene, M. Somayazulu, E. Vinitsky, D. Chandra, T. A. Strobel, V. V. Struzhkin, and R. J. Hemley, J. Phys. Chem. C 119, 18007 (2015).

[39] M. Rahm, R. Hoffmann, and N. W. Ashcroft, J. Am. Chem. Soc. 139, 8740 (2017).

[40] F. Tian, D. Li, D. Duan, X. Sha, Y. Liu, T. Yang, B. Liu, and T. Cui, Mater. Res. Express 2, 046001 (2015).

[41] M.-s. Miao, X.-1. Wang, J. Brgoch, F. Spera, M. G. Jackson, G. Kresse, and H.-q. Lin, J. Am. Chem. Soc. 137, 14122 (2015).

[42] J. Lin, S. Zhang, W. Guan, G. Yang, and Y. Ma, J. Am. Chem. Soc. 140, 9545 (2018).
[43] J. Botana, X. Wang, C. Hou, D. Yan, H. Lin, Y. Ma, and M.-s. Miao, Angew. Chem. Int. Ed. 54, 9280 (2015).

[44] J. Lin, Z. Zhao, C. Liu, J. Zhang, X. Du, G. Yang, and Y. Ma, J. Am. Chem. Soc. 141, 5409 (2019).

[45] Y. Li, J. Hao, H. Liu, Y. Li, and Y. Ma, J. Chem. Phys. 140, 174712 (2014).

[46] Y. Li, L. Wang, H. Liu, Y. Zhang, J. Hao, C. J. Pickard, J. R. Nelson, R. J. Needs, W. Li, Y. Huang et al., Phys. Rev. B 93, 020103(R) (2016).

[47] I. Kruglov, R. Akashi, S. Yoshikawa, A. R. Oganov, and M. M. D. Esfahani, Phys. Rev. B 96, 220101(R) (2017).

[48] A. P. Drozdov, M. I. Eremets, I. A. Troyan, V. Ksenofontov, and S. I. Shylin, Nature (London) 525, 73 (2015).

[49] K. Abe and N. W. Ashcroft, Phys. Rev. B 84, 104118 (2011).

[50] Y. Yao and R. Hoffmann, J. Am. Chem. Soc. 133, 21002 (2011).

[51] A. M. Murcia Rios, D. N. Komsa, and V. N. Staroverov, J. Phys. Chem. C 122, 14781 (2018).

[52] C. Murli and Y. Song, J. Phys. Chem. B 113, 13509 (2009).

[53] A. Torabi, Y. Song, and V. N. Staroverov, J. Phys. Chem. C 117, 2210 (2013).

[54] A. Torabi, C. Murli, Y. Song, and V. N. Staroverov, Sci. Rep. 5, 13929 (2015).

[55] Y. Song, C. Murli, and Z. Liu, J. Chem. Phys. 131, 174506 (2009).

[56] C.-H. Hu, A. R. Oganov, Q. Zhu, G.-R. Qian, G. Frapper, A. O. Lyakhov, and H.-Y. Zhou, Phys. Rev. Lett. 110, 165504 (2013).

[57] W.-H. Yang, W.-C. Lu, S.-D. Li, X.-Y. Xue, Q.-J. Zang, K. M. Ho, and C. Z. Wang, Phys. Chem. Chem. Phys. 21, 5466 (2019).

[58] B. Krebs and H.-U. Hürter, Angew. Chem. 92, 479 (1980).

[59] H. Wu, X. Li, R. Zhang, and J. Yang, J. Mater. Chem. A 7, 3752 (2019).

[60] B. Mortazavi and T. Rabczuk, Energies 11, 1573 (2018).

[61] H. Diercks and B. Krebs, Angew. Chem., Int. Ed. Engl. 16, 313 (1977).

[62] D. Fan, C. Yang, S. Lu, and X. Hu, arXiv:1803.03459.

[63] T. Sasaki, H. Takizawa, K. Uheda, T. Yamashita, and T. Endo, J. Solid State Chem. 166, 164 (2002).

[64] K. A. Cherednichenko, I. A. Kruglov, A. R. Oganov, Y. Le Godec, M. Mezouar, and V. L. Solozhenko, J. Appl. Phys. 123, 135903 (2018).

[65] T. Sasaki, H. Takizawa, K. Uheda, and T. Endo, Phys. Status Solidi B 223, 29 (2001).

[66] Y. Wang, J. Lv, L. Zhu, and Y. Ma, Phys. Rev. B 82, 094116 (2010).

[67] Y. Wang, J. Lv, L. Zhu, and Y. Ma, Comput. Phys. Commun. 183, 2063 (2012).

[68] A. R. Oganov and C. W. Glass, J. Chem. Phys. 124, 244704 (2006).

[69] C. J. Pickard and R. J. Needs, J. Phys.: Condens. Matter 23, 053201 (2011).

[70] S. Curtarolo, G. L. W. Hart, M. B. Nardelli, N. Mingo, S. Sanvito, and O. Levy, Nat. Mater. 12, 191 (2013).

[71] L. Zhang, Y. Wang, J. Lv, and Y. Ma, Nat. Rev. Mater. 2, 17005 (2017).

[72] J. Lv, Y. Wang, L. Zhu, and Y. Ma, Phys. Rev. Lett. 106, 015503 (2011).

[73] L. Zhu, H. Wang, Y. Wang, J. Lv, Y. Ma, Q. Cui, Y. Ma, and G. Zou, Phys. Rev. Lett. 106, 145501 (2011).

[74] H. Wang, J. S. Tse, K. Tanaka, T. Iitaka, and Y. Ma, Proc. Natl. Acad. Sci. USA 109, 6463 (2012). 
[75] See Supplemental Material at http://link.aps.org/supplemental/ 10.1103/PhysRevB.100.134110 for computational details, the convex hull of B-S system between $1 \mathrm{~atm}$ to $200 \mathrm{GPa}$, structural information, phonon dispersion curves, electronic properties, superconductive properties, and Bader charge analysis of B-S phases.

[76] P. Hohenberg and W. Kohn, Phys. Rev. 136, B864 (1964).

[77] W. Kohn and L. J. Sham, Phys. Rev. 140, A1133 (1965).

[78] G. Kresse and J. Furthmüller, Phys. Rev. B 54, 11169 (1996).

[79] J. P. Perdew, K. Burke, and M. Ernzerhof, Phys. Rev. Lett. 77, 3865 (1996).

[80] J. P. Perdew, J. A. Chevary, S. H. Vosko, K. A. Jackson, M. R. Pederson, D. J. Singh, and C. Fiolhais, Phys. Rev. B 46, 6671 (1992).

[81] P. E. Blöchl, Phys. Rev. B 50, 17953 (1994).

[82] H. J. Monkhorst and J. D. Pack, Phys. Rev. B 13, 5188 (1976).

[83] A. Togo, F. Oba, and I. Tanaka, Phys. Rev. B 78, 134106 (2008).

[84] P. Giannozzi, S. Baroni, N. Bonini, M. Calandra, R. Car, C. Cavazzoni, D. Ceresoli, G. L Chiarotti, M. Cococcioni, I. Dabo et al., J. Phys.: Condens. Matter 21, 395502 (2009).
[85] L. Zhu, H. Liu, R. E. Cohen, R. Hoffmann, and T. A. Strobel, arXiv:1708.03483.

[86] C. J. Pickard and R. J. Needs, Nat. Phys. 3, 473 (2007).

[87] A. R. Oganov, J. Chen, C. Gatti, Y. Ma, Y. Ma, C. W. Glass, Z. Liu, T. Yu, O. O. Kurakevych, and V. L. Solozhenko, Nature (London) 457, 863 (2009).

[88] Y. Akahama, M. Kobayashi, and H. Kawamura, Phys. Rev. B 48, 6862 (1993).

[89] H. Luo, R. G. Greene, and A. L. Ruoff, Phys. Rev. Lett. 71, 2943 (1993).

[90] G. Yang, S. Shi, J. Yang, and Y. Ma, J. Mater. Chem. A 3, 8865 (2015).

[91] J. Heyd, G. E. Scuseria, and M. Ernzerhof, J. Chem. Phys. 118, 8207 (2003).

[92] A. V. Krukau, O. A. Vydrov, A. F. Izmaylov, and G. E. Scuseria, J. Chem. Phys. 125, 224106 (2006).

[93] A. D. Becke and K. E. Edgecombe, J. Chem. Phys. 92, 5397 (1990).

[94] R. F. W. Bader, Acc. Chem. Res. 18, 9 (1985).

[95] D. Duan, Y. Liu, F. Tian, D. Li, X. Huang, Z. Zhao, H. Yu, B. Liu, W. Tian, and T. Cui, Sci. Rep. 4, 6968 (2014). 